\title{
Review of: "Archaeometric studies on a Pompeian blue glass fragment from Regio I, Insula 14 for the characterization of glassmaking technology"
}

\author{
Vladimir Ivanovski \\ Potential competing interests: The author(s) declared that no potential competing interests exist.
}

\section{Dear Research Square}

I was asked to review the manuscript under the title: "Archaeometric studies on a Pompeian blue glass fragment from Regio I, Insula 14 for the characterization of glassmaking technology". Below are the results of my review.

The manuscript opens an interesting question whether the presence of the primary glass production industry and a possible Pompeian secondary workshop for the production of glass manufactures during the 1st century AD existed. Authors' idea is through chemical analysis of a Pompeian blue glass fragment, to seek for the origin of the raw materials used for the production of the glass, particularly the sand. Authors used several techniques like light microscope, Scanning Electron Microscope, IR and Raman spectroscopy and ICP-MS.

Since my field of expertise is IR and Raman spectroscopy, my questions and remarks will be primarily from that part of the manuscript.

1. On page 4. "Reflection spectra (200 acquisitions) were collected using the microscope focusing windows set at 50x50 mm."

Q1. What is the incidence angle, or maybe the average incidence angle?

2. On page 6. "Raman spectrum of the sample, reported in Fig. 3a, highlighted the presence of two major peaks at 1090 and $584 \mathrm{~cm}-1$ with and two well-defined components at 945 and $995 \mathrm{~cm}-1 . "$

Q2. What is the origin of the components at 945 and $995 \mathrm{~cm}-1$ ? Are they result of the splitting of the mode or the type of bridging in the $\mathrm{SiO} 4$ entity?

Q2.1. Why these bands are not taken into the fitting procedure (cf. Fig. 3a). I understood they should be, according to the here presented references and (Colomban Ph. J. Non-Crystalline Solids 2003; 322: 180.), where the area of the envelopes at ca.500 and ca.1000 cm-1 bands are being calculated, and not only one of the components. 
3. On page 6. "According to this classification, the Ip value calculated from Raman spectra collected in a different area of the sample $(I p=0.6 \pm 0.05)$ would correspond to a family of silicate-based glasses characterized..."

Q3. Why on fig. 4 the baseline is below zero? Do Authors think integration will give correct values if this is the case? Why no convolution and no base line subtraction was performed, as explained in the cited literature? The fitting of the Raman spectrum seems to me a bit poor, visually, since there is no quantitative value of how good the fitting is.

4. On page 6. "The spectrum was characterized by bands at $2926-2844 \mathrm{~cm}-1$ (functional groups region) and 1725-1586 cm-1 (double bond stretching) regions because of $\mathrm{C}-\mathrm{H}$ bending [36, 37]..."

Q4. This sentence should be rewritten. What is the connection of the C-H bending with those regions? In my opinion there is no.

5. In Table 2. "3590 Symmetric stretching of O-H groups (vO-H). Silanol group (Si-OH)."

Q5. I doubt that only one wavenumber can characterise the $\mathrm{OH}$ stretching of this complex of bands (as authors mention in the text, a broad band). It is better to use a range of wavenumbers in this case.

6. Figure 3b. IR absorbance vs wavenumber

Q6. In the experimental part authors say they employed IR specular reflectance technique. What was the way of converting the reflectance, into absorbance spectrum? It must be stressed that reflectance and absorbance spectra IR spectra look quite different, particularly that reflectance spectra are quite dependent on the incidence angle; here saying that reflectance is not a transmittance that can be easily converted into absorbance. For that one would need a Kramers-Kronig relations or dispersion analysis.

There are some other minor remarks, like some typing mistakes present, maybe also strengthening the language, too. Also, assignments in Table 2 need to be clearly presented. For example, Authors write about carbonate stretching and there is no frequency assigned nor carbonate as an inion is of question in this case, as I understood.

Overall, I think the manuscript describes interesting subject and gives valuable information, but Authors should address the above stated question, and in particularly Q2.1, Q3 and Q6. 